A N N A L E S Annales de Bretagne et des Pays de l'Ouest

Anne Jollet, Terre et société en Révolution. Approche du lien social dans la région d'Amboise

\title{
Brigitte Maillard
}

\section{(2) OpenEdition}

1 Journals

\section{Édition électronique}

URL : http://journals.openedition.org/abpo/1755

DOI : $10.4000 / a b p o .1755$

ISBN : 978-2-7535-1482-9

ISSN : 2108-6443

\section{Éditeur}

Presses universitaires de Rennes

Édition imprimée

Date de publication : 20 juin 2001

Pagination : 167-170

ISBN : 978-2-86847-635-7

ISSN : 0399-0826

\section{Référence électronique}

Brigitte Maillard, « Anne Jollet, Terre et société en Révolution. Approche du lien social dans la région d'Amboise ", Annales de Bretagne et des Pays de l'Ouest [En ligne], 108-2 | 2001, mis en ligne le 20 juin 2003, consulté le 23 septembre 2020. URL : http://journals.openedition.org/abpo/1755 ; DOI : https:// doi.org/10.4000/abpo. 1755 
Anne Jollet, Terre et société en Révolution. Approche du lien social dans la région d'Amboise, Paris, Éditions du CTHS, 2000, 548 p., 250 F.

Dans sa thèse, dont elle présente ici une version remaniée, A. Jollet a choisi d'étudier le "lien social " dans une "micro-région" (soit deux cantons révolutionnaires, $260 \mathrm{~km}^{2}$ et 4000 feux, environ 15500 habitants), située dans la vallée de la Loire, avec au centre sa petite capitale, Amboise (5000 habitants environ); l'unité économique de ce secteur est due a la présence du vignoble dont les produits peuvent être exportés facilement grâce à la Loire. Pour étudier le "vivre ensemble ", A. Jollet a adopte comme méthode l'utilisation systématique d'archives dont on n'ignore pas l'intérêt pour l'histoire économique (cf. les études de G. Béaur) mais dont la massivité et la "sécheresse " exercent parfois un effet repoussoir. L'auteur a en effet privilégié pour une étude sociale des archives économiques, tout particulièrement les données sur les mutations foncières fournies par le Contrôle des Actes/Enregistrement et les dossiers de la vente des Biens Nationaux. La période étudiée s'étend de 1780 à 1816, temps bref mais marqué par le choc de la mise en vente des Biens Nationaux; la situation en 1780 et en 1811 est plus particulièrement scrutée mais des coupes chronologiques intermédiaires permettent de préciser les étapes et les rythmes de l'évolution à partir du tableau dressé pour les années 1780. Ce dernier s'appuie en outre sur l'utilisation des rôles de taille heureusement conservés (exception unique en Touraine) pour l'élection d'Amboise, de certaines sources notariales comme les quittances (mais le problème est que ces actes en brevet ne sont pas tous conservés par les notaires et que tous les paiements ne donnent pas lieu à la rédaction d'un tel acte) ainsi que sur les cahiers de doléances ; ainsi est dressé un tableau de différentes relations sociales dans les années qui précèdent la Révolution.

A. Jollet a fait sienne l'idée de G. Lefevbre selon laquelle « il ne suffit pas de décrire, encore faut-il compter "; elle a donc soumis son corpus documentaire à un traitement statistique simple mais serré et elle fournit de très abondantes données chiffrées, le plus souvent sous la forme de fréquences qui mettent en rapport, selon toutes les approches croisées possibles, vendeurs et acheteurs, superficies, qualités, situations des biens concernés; mais malgré le nombre impressionnant d'actes dépouillés, il arrive que l'échantillon étudié soit mince et soumis à des variations statistiques aléatoires. II faut regretter que les contraintes éditoriales l'aient amenée à supprimer de nombreux tableaux qui auraient aéré un texte très dense et donné une plus grande lisibilité à des résultats qui doivent être présentés dans le texte et perdent en possibilités de comparaisons. Plus que des liens sociaux et économiques durables, ce sont des relations brèves, le temps des tractations menées pour réaliser un transfert de propriété immobilière ou foncière, qui sont étudiées, dans le cadre du marché ordinaire ou des opérations exceptionnelles que représente la vente 
des Biens Nationaux. Au-delà se dessinent des politiques foncières, propres à chacun des groupes sociaux.

Dans la définition de ces derniers les problèmes de méthode ne sont pas éludés ; parler des groupes sociaux pose la question de la qualification des individus, en particulier des ruraux; à l'intérieur même du groupe des vignerons les situations économiques peuvent être très contrastées; chez les autres paysans, les laboureurs sont bien distingués mais entre ces derniers et les journaliers les mots manquent pour désigner les situations intermédiaires. Les rôles de taille, qui peuvent sembler les documents les plus précis et les plus cohérents, présentent l'inconvénient de n'accorder qu'une attention limitée aux plus pauvres et de s'occuper fort peu des privilégiés. Enfin la situation des femmes (veuves et filles) est la plus ambiguë; sans cacher les inconvénients d'un tel choix, A. Jollet en fait une catégorie à part, qui semble se définir par sa position par rapport aux hommes, mais constatant la grande diversité des situations, elle sait réintégrer parfois les veuves dans leur groupe d'appartenance sociale. Un autre problème redoutable est celui de l'estimation à donner de la valeur exacte des biens vendus puisque celle-ci est fonction de très nombreux facteurs, comme la diversité de l'utilisation (les prés l'emportent toujours en valeur), de la superficie (les lopins isolés sont proportionnellement beaucoup plus coûteux que les parcelles incluses dans une exploitation), la présence du bâti, le poids des rentes ou de droits seigneuriaux...

La comparaison entre les transactions immobilières et foncières qui ont lieu dans les décennies 1780 et 1810 montre une amplification et une accélération très fortes du rythme des ventes : en 1780 elles touchent 210 ha pour une valeur de $222000 \mathrm{~L}$. contre 490 ha valant 695 000 francs en 1811, mais si les surfaces concernées ont été multipliées par 2,3 le nombre des articles l'a été par 3,7, c'est à dire que la taille des parcelles a encore diminué. Les périodes plus particulièrement marquées par l'augmentation sont 1786-1791, 1796-1803 où le nombre de transactions triple ou presque, puis 1803-1811 mais avec un rythme plus lent. Les acheteurs sont plus nombreux que les vendeurs. Dans la recherche des facteurs d'explication de cette " mise en mouvement " que connaît le marché foncier, l'effet d'accélération dû à la vente des biens nationaux apparaît très nette : pour cette région qui n'avait pas encore été étudiée, A. Jollet met en évidence l'étendue de la propriété du clergé régulier et son organisation bipolaire : l'Église détient de très nombreuses parcelles dont les prix sont accessibles pour des laboureurs ou des vignerons aisés, ainsi que des closeries et des métairies qui, une fois vendues, n'échapperont plus aux partages successoraux et passeront ainsi plus facilement dans le marché. Enfin dans quelques paroisses, la noblesse possède des surfaces importantes, même si on ne tient pas compte de la présence du domaine de Chanteloup qui a appartenu à Choiseul, puis aux Penthièvre. 
Si les mouvements du marché foncier du côté des vendeurs comme des acheteurs (pris en compte à chaque intervention, ou " occurrence ") sont étudiés sous tous leurs aspects (économiques, financiers, sociaux), les structures foncières s'en trouvent aussi mises en évidence : la parcellisation du sol, quelle que soit la nature de l'occupation de la terre, est forte et cet émiettement facilite l'accès à la propriété des petits paysans, en particulier des vignerons. Le parcellaire connaît une double évolution pendant la période étudiée : à la fois la poursuite de l'émiettement dû en grande partie aux partages successoraux mais aussi la constitution de parcelles d'une superficie plus importante et qui trouvent leur place entre les petits lopins et les exploitations constituées (closeries et métairies). Ces dernières restent toujours aussi petites et leur propriété échappe aux paysans, avant comme après la Révolution. En effet, entre 1780 et 1811, globalement la domination des catégories les plus aisées n'est pas remise en cause ; la propriété foncière de la bourgeoisie fait des progrès, mais la bourgeoisie marchande et manufacturière locale voit sa part se réduire au profit des "rentiers "; la part du clergé baisse évidemment. Quant à la noblesse, elle connaît un retour des anciennes familles (comme celui des Martigny à Nazelles) mais aussi un réel renouvellement elle conserve son emprise sur de vastes superficies : le domaine de Chanteloup (593 ha) est acheté en 1795 par le parisien Lefrançois, et quelques années après par Chaptal. Le poids global de la ville diminue mais les achats urbains portent sur de grosses surfaces. Les paysans aisés et moyens, comme les vignerons, sont très présents tandis que journaliers et fagoteurs continuent à se glisser rarement parmi les acheteurs. Toutefois, la vente des biens de seconde origine a été moins favorable aux ruraux que celle des biens de première origine, contrairement à ce qui a pu être observé pour le district de Tours. À la fin de l'Empire, le phénomène de proximité s'affaiblit et les interventions extérieures sont plus nombreuses : des habitants de Tours et de Paris interviennent; de même, si les paysans continuent à traiter de préférence avec d'autres paysans leurs partenaires se diversifient. La société ne se trouve pas bouleversée mais la place du groupe des ruraux dans la propriété se trouve accrue. Malheureusement les sources ne permettent pas de connaître les situations individuelles et de savoir si cette ouverture du marché foncier a permis l'accès à la propriété foncière de nombreux individus ou de seulement conforter des situations déjà acquises.

Dans cette étude d'un marché foncier et de ses acteurs parmi lesquels des artisans comme les tonneliers occupent une place non négligeable, une réalité économique est bien mise en valeur, celle de la médiocrité du vignoble amboisien à la fin du XVIII et au début du XIX ${ }^{\mathrm{e}}$ siècle; il n'est pas un placement recherché et ses nombreux lopins n'attirent pas d'acheteurs autres que les vignerons qui n'ont pas les moyens d'acquérir des closeries 
Étant donné le volume du texte, les coquilles sont rares ; il vaudrait mieux toutefois que les patronymes des familles nobles (par exemple dans le cas de la famille Sain de Bois le Comte) soient transcrits d'une manière identique dans tout le texte; I'abbé Chevalier n'est pas l'auteur du Rapport sur la généralité de Tours 1762-1766 mais il en a édité la partie concernant la Touraine. Quelques formules gagneraient à être nuancées; les biens d'Église ne sont pas inaliénables (p. 403) et les nouvelles acquisitions sont possibles mais soumises a l'obtention d'une permission royale.

Pour aller plus loin dans ce qu'A. Jollet elle-même appelle une " approche du lien social ", des recherches pourraient être menées à partir d'autres archives notariales, comme les contrats de location ou les rapports maîtres/serviteurs, qui tout en restant dans le registre économique, permettraient d'approcher des relations qui permettent de tisser des liens, sans doute plus conflictuels, mais plus durables dans ce petit monde.

Brigitte Maillard

Michèle COINTET, L'Église sous Vichy 1940-1945. La repentance en question, Librairie académique Perrin, 1998, 404 p.

Même si son sujet n'est plus vraiment neuf, comme l'avait souligné Étienne Fouilloux dans Les chrétiens français entre crise et libération 1937-1947 (Le Seuil, 1997) compte tenu de l'abondance de l'historiographie le concernant, cet ouvrage est dense tout en étant accessible à un large public. Le titre est un peu réducteur en ce sens qu'il ne traite pas seulement de l'Église en tant qu'institution hiérarchique, de ses attitudes et réactions pendant l'Occupation. Certains chapitres décrivent par le menu la politique religieuse du gouvernement de Vichy, que M. Cointet avait déjà abordée dans sa thèse sur le Conseil national (en particulier quant à la question de la jeunesse) et la synthèse se complète d'aperçus sur les attitudes contrastées des catholiques (avec des ouvertures sur les protestants) devant la Collaboration et la Résistance.

Le changement de régime, en 1940, a représenté une bénédiction pour l'Église catholique. En rupture avec la III ${ }^{\mathrm{e}}$ République dont l'anticléricalisme était certes bien atténué, I'État français prétendait promouvoir des valeurs qui rejoignaient les valeurs religieuses traditionnelles, dont l'oubli était perçu comme un des facteurs de la défaite, porter un projet de société conforme à la doctrine sociale des encycliques pontificales, s'appuyer sur l'Église dont il recherchait la caution. Le corps épiscopal, largement renouvelé dans l'entre-deuxguerres, avait dans sa majorité fait l'expérience de la mobilisation pendant la Première Guerre mondiale et la sympathie pour le vainqueur 